\title{
QUANTIZATION OF CHEMICAL REACTION: THE DYNAMIC CORRELATION DIAGRAM METHOD FREE FROM NONCROSSING RULE
}

\author{
HIROYUKI NOHIRA* \\ Department of Applied Chemistry \\ Faculty of Engineering, Saitama University \\ Shimo-okubo 255, Sakura-ku \\ Saitama 338-8570, Japan \\ h.nohira@jcom.home.ne.jb \\ TOSHIYUKI NOHIRA \\ Department of Fundamental Energy Science \\ Graduate School of Energy Science \\ Kyoto University, Sakyo-ku \\ Kyoto 606-8501, Japan \\ nohira@energy.kyoto-u.ac.jp
}

Received 19 June 2011

Accepted 13 September 2011

\begin{abstract}
Both Fukui's frontier orbital (FO) theory and Woodward-Hoffmann's orbital symmetry conservation $(\mathrm{W}-\mathrm{H})$ theory are based on the molecular orbital theory. However, there are some obvious inconsistencies between the two theories in explaining the electron movement. The process of chemical reactions has been explained by the potential surface analysis based on the time-independent Schrödinger equation. However, this approach is not always appropriate for describing unsteady states, because the variable of reaction coordinate should be time $t$ by its very nature. When considering the time-scale of chemical reactions for molecules, there is inherent uncertainty in the energy levels for the midway state of chemical reactions owing to the Heisenberg uncertainty principle. The states which can be accurately described by quantum mechanics exist discontinuously in chemical reactions. Such quantization of chemical reactions solves all noncrossing problems. We also show that such an essential fact leads to new concepts and theories in chemical reactions such as stable molecule, elementary reaction and minimum deformation of orbital phases. Finally, taking the regularity in organic reactions as an example, we demonstrate that $\mathrm{FO}$ theory and $\mathrm{W}-\mathrm{H}$ theory can be unified consistently by the universally applicable "dynamic correlation diagram method."
\end{abstract}

Keywords: Quantum chemical dynamics; Heisenberg uncertainty principle; noncrossing rule; elementary reaction; minimum deformation of orbital phase; correlation diagram.

*Current address: Saitama Research Laboratory, 51-5 Okubo-ryoke, Sakura-ku, Saitama 338-0826, Japan.

This is an Open Access article published by World Scientific Publishing Company. It is distributed under the terms of the Creative Commons Attribution 4.0 (CC-BY) License. Further distribution of this work is permitted, provided the original work is properly cited. 


\section{Introduction}

Fukui and Hoffman were awarded a Nobel Prize in 1981 for their pioneering theories which explain the regularity in organic reactions. ${ }^{1-6}$ However, there are obvious inconsistencies between frontier orbital (FO) theory and Woodward-Hoffmann's $(\mathrm{W}-\mathrm{H})$ correlation diagram method, e.g., a contradiction of the electron movement in explaining the Diels-Alder reaction of butadiene with ethylene. ${ }^{2,5-7}$ Moreover, it has been pointed out that although orbital correlation diagrams, regarded as an advanced application of the diatomic correlation diagram by Hund ${ }^{8}$ and Mulliken ${ }^{9}$ to organic reactions, are the foundation of $\mathrm{W}-\mathrm{H}$ rules, proper orbital correlation diagrams cannot be drawn for many reactions by W-H's method. ${ }^{10}$

In 1974, we proposed that FO theory and $\mathrm{W}-\mathrm{H}$ theory could be unified consistently by introducing a new, simple concept of minimum deformation of orbital phases. ${ }^{11}$ This concept also enables the construction of appropriate orbital correlation diagrams without exception. ${ }^{11}$ Unfortunately, our concept was criticized $^{12,13}$ because it disregards the noncrossing rule proposed by von Neumann and Wigner. ${ }^{14}$ In response to the criticisms, we insisted ${ }^{15,16}$ that the noncrossing rule is based on the Born-Oppenheimer (BO) approximation (adiabatic approximation), ${ }^{17}$ and that the applicability of the noncrossing rule has a limitation due to the Heisenberg uncertainty principle ${ }^{18}$ in quantum mechanics. Yet, our concept remains unaccepted to this day, probably owing to traditional beliefs in both the BO approximation and the noncrossing rule.

Recently, however, nonadiabatic transition ${ }^{19}$ has attracted much attention in the field of quantum chemistry and the limitation of the BO approximation has been pointed out. ${ }^{20,21}$ Such non-BO quantum chemistry has indicated that the noncrossing problem is explained by the concept of "natural correlation or natural crossing," $22-25$ "conical intersection" ${ }^{26-30}$ and/or "nonadiabatic transition." 20 However, when considering the time-scale of chemical reactions for molecules, there is inherent uncertainty in the energy levels for the midway state of chemical reactions owing to the Heisenberg uncertainty principle. Here we show, in a plain and thorough way for the first time, conclusive answers to all the noncrossing problems and all the inconsistencies between FO theory and $\mathrm{W}-\mathrm{H}$ theory. We also demonstrate the universal applicability of "the dynamic correlation diagram method."

\section{Theoretical Basis}

\subsection{Limitation of time-independent Schrödinger equation}

First of all, we have to recall the basis of molecular orbital (MO) theory. The most general form of the Schrödinger equation is time dependent; it describes how the quantum state of a physical system changes as a function of position, $\boldsymbol{r}$, and time, $t .{ }^{31-34}$

$$
i \hbar \frac{\partial \Psi(\mathbf{r}, t)}{\partial t}=\left\{-\frac{\hbar^{2}}{2 m} \nabla^{2}+V(\mathbf{r}, t)\right\} \Psi(\mathbf{r}, t) .
$$


Although this equation cannot be solved in this form, it can be modified by the following assumptions:

(a) Separation of variables $\boldsymbol{r}$ and $t$

$$
\Psi(\boldsymbol{r}, t)=\Psi(\boldsymbol{r}) \cdot \exp \left(-\frac{i E t}{\hbar}\right) .
$$

(b) Stationary-state approximations such as BO approximation. ${ }^{18}$

The resulting time-independent Schrödinger equation ${ }^{35}$ is

$$
E \Psi(\boldsymbol{r})=\left\{-\frac{\hbar^{2}}{2 m} \nabla^{2}+V(\boldsymbol{r})\right\} \Psi(\boldsymbol{r}) .
$$

Quantum chemistry, including MO theory, has obviously been developed on the basis of the time-independent Schrödinger equation. Although this science is extremely powerful for stationary states, the theories and calculations derived from the time-independent Schrödinger equation are not always appropriate for describing unsteady states, such as a transition state in elementary reactions. For instance, in quantum chemical dynamics, most studies on reaction paths have been performed by the analysis of potential energy surface calculated by the time-independent Schrödinger equation. However, no consideration has been paid for the time scale of the reaction, in spite of the fact that the variable of reaction coordinate should be time $t$ by its very nature. The following is a simple calculation method to estimate the minimum time-scale which can be used for the time-independent Schrödinger equation. In formula (2), which is a premise for formula (3), the time term, $\exp (-i E t / \hbar)$, is equal to $\cos (E t / \hbar)-i \sin (E t / \hbar)$, and is obviously a periodic function determined by $E$. If $E$ is assumed to be $0.43 \mathrm{eV}\left(10 \mathrm{kcal} \mathrm{mol}^{-1}, 41.8 \mathrm{~kJ}\right.$ $\mathrm{mol}^{-1}$ ), for example, then the period of vibration, $T$, is calculated as $0.94 \times 10^{-14} \mathrm{~s}$. Obviously, the time-independent Schrödinger equation is valid for the states which exist longer than their own vibration periods.

\subsection{Validity of noncrossing rule in chemical reactions}

One typical problem which should be reexamined is the noncrossing rule formulated by von Neumann and Wigner in 1929. ${ }^{14}$ It states that energy levels corresponding to electronic states of the same symmetry cannot cross. In 1936, Teller has suggested ${ }^{36}$ that for the case of polyatomic molecules where, in general, more than one parameter is necessary to describe the reaction process, the possibility of the crossing of energy surfaces cannot be excluded because their electronic states are identical in symmetry properties. This concept has been reconfirmed by Herzberg and LonguetHiggins $^{37}$ : the noncrossing rule is valid only for diatomic molecules, while for polyatomic molecules this rule is not valid. This seems to be accepted fairly generally today. It is, however, worth noting that Longuet-Higgins himself and Abrahamson $^{38}$ first applied the correlation diagram method to the organic reactions in connection with the $\mathrm{W}-\mathrm{H}$ theory, where the noncrossing rule was observed. 
Since then, almost all the correlation diagrams for the $\mathrm{W}-\mathrm{H}$ theory have been drawn so as not to violate the noncrossing rule. ${ }^{39-43}$

The noncrossing rule is mathematically correct as long as the time-independent Schrödinger equation is used. On the other hand, for systems in which the quantum state changes with time, such as chemical reactions, the noncrossing rule needs reconsideration. Conventional approaches to the noncrossing problem are the introduction of the non-BO approximation and/or time-dependent Schrödinger equation. However, there is inherent uncertainty in the energy levels for the midway states of chemical reactions owing to the Heisenberg uncertainty principle. ${ }^{18}$

$$
\Delta E \cdot \Delta t \geq \frac{\hbar}{2}
$$

This principle clearly states "a state that only exists for a short time cannot have a definite energy." Illustration using an actual calculation will be informative. Since all atoms and molecules have certain characteristic vibration periods, typically, on the order of 10 to $100 \mathrm{fs}\left(1 \mathrm{fs}=10^{-15} \mathrm{~s}\right)$, it is reasonable to consider that elementary reactions proceed within this period of vibration. ${ }^{15,44}$ Here we assume that an elementary reaction occurs in approximately $5 \times 10^{-14} \mathrm{~s}$. Figure 1 shows a schematic of the potential energy surface for an elementary reaction. In a conventional potential energy surface, a geometric parameter such as bond length and bond angle is taken as a reaction coordinate $(r)$, and time $(t)$ is not considered. However, for elementary reactions, $r$ should be a function of $t$ because the state changes with time. Therefore, it is possible and reasonable to take time as the reaction coordinate. This point becomes especially important when applying the potential energy surface to quantum chemical dynamics. For a simplified argument, in Fig. 1, we assume that $r$ is expressed so that it becomes proportional to change in $t$. If we divide the reaction process into 10 equal sections, it takes $5 \times 10^{-15} \mathrm{~s}$ to pass through one section. If any midway state, to which the noncrossing rule has been applied, is included in one section, the energy uncertainty is calculated to be larger than

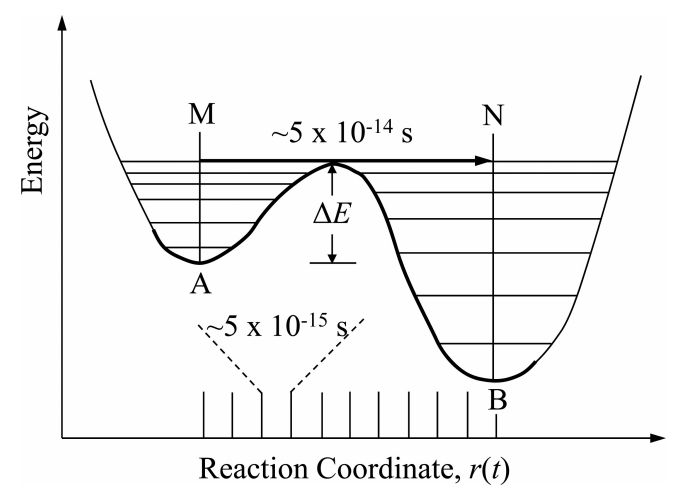

Fig. 1. A schematic representation of the potential energy surface and time course for an elementary reaction. 
$6.3 \mathrm{~kJ} \mathrm{~mol}^{-1}$ by substituting $5 \times 10^{-15} \mathrm{~s}$ for $\Delta t$ in Eq. (4). Under these circumstances, the noncrossing rule is no longer valid, even in the case of diatomic molecules. The importance of this self-evident fact has long been ignored.

\subsection{New definitions of stable molecules and elementary reactions}

As a natural consequence of the above considerations, the following definitions are possible for the terms "stable molecules" and "elementary reactions." First, a "stable molecule" can denote a group of atoms having a lifetime of at least one characteristic vibration. Clearly, a quantum chemical calculation possesses a practical physical meaning only for such stable molecules. An "elementary reaction" can be defined as a one-step reaction that does not have any stable intermediate molecules (or stable activated complexes). If a certain intermediate has a lifetime longer than one vibration period, it should be regarded as a stable molecule and a new elementary reaction can be formulated. Consequently, the passage through a transition state always occurs within a considerably shorter time than one vibration period.

\section{The Dynamic Correlation Diagram Method}

Since we are no longer restrained by the noncrossing rule, we propose the new concept of dynamic correlation diagrams that are simply drawn according to the idea of minimum deformation of molecular orbitals, which assume that the change of molecular orbital in elementary reactions proceeds with the least deformation. This idea is an application of the variational principle or the principle of least action, the most general and practical approach in physics. In 1965, Fano and Lichten ${ }^{45}$ proposed a correlation diagram of diabatic molecular orbitals for inelastic $\mathrm{Ar}^{+}-\mathrm{Ar}$ collisions, in which energy level crossing occurs. Our dynamic correlation diagram can be regarded as an advanced application of the Fano-Lichten correlation diagram to organic reactions. The typical examples of dynamic correlation diagrams given below (see Figs. 2-4) will help to illustrate this new concept.

These correlation diagrams have been drawn in the same style used by Woodward and Hoffmann ${ }^{5,6}$ : On one side, one writes down the calculated approximate energy levels of the orbitals for the reactants, and on the other side, those for the products. Since high accuracy is not required, the simple Hückel method ${ }^{46,47}$ has been used for the calculation of energy levels. The phases of wave functions are color-coded: Positive $=$ blue , Negative $=$ green or vice versa . The molecular symmetry is denoted as follows: $\mathrm{S}=$ symmetric, $\mathrm{A}=$ antisymmetric.

\subsection{The Diels-Alder reaction of butadiene with ethylene}

Figure 2 compares correlation diagrams for the Diels-Alder reaction of butadiene with ethylene proposed by the present authors (a dynamic correlation diagram, solid lines) and Woodward-Hoffmann (dashed lines). ${ }^{5,6}$ In our diagram, all the 

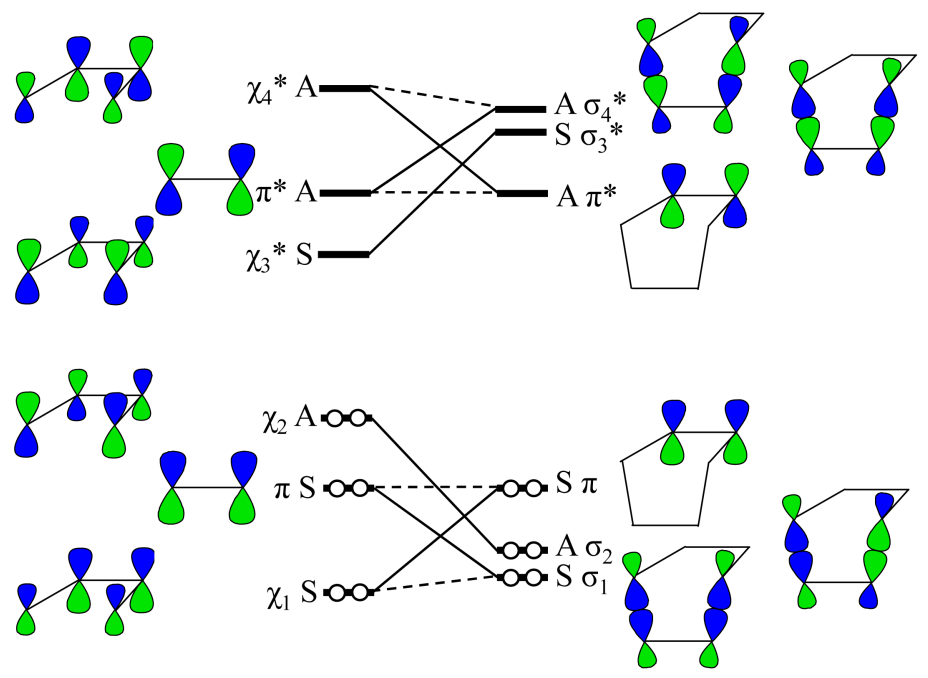

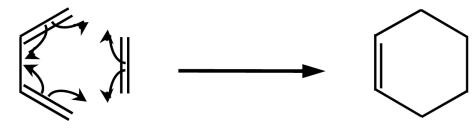

Scheme 1

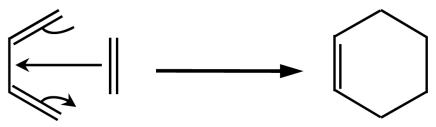

Scheme 2

Fig. 2. Correlation diagrams for the Diels-Alder reaction of butadiene with ethylene proposed by the present authors (a dynamic correlation diagram, solid lines) and Woodward-Hoffmann ${ }^{5,6}$ (dashed lines). Schemes 1 and 2 are derived from the solid line and the dashed line diagrams, respectively.

correlation lines are simply drawn to minimize the deformation of molecular orbital phases, such as symmetries, patterns or shapes, between the reactants and the product. The $\chi_{1}$ orbital of butadiene is connected with the $\pi$ orbital of cyclohexene, and the $\pi$ orbital of ethylene is linked with the $\sigma_{1}$ orbital of cyclohexene. As a result, there is a "crossing" of two correlation lines connecting the electronic states of the same symmetry. This diagram clearly shows that the electron movement is explained by Scheme 1: the $\pi$ bond of cyclohexene is formed by the $\chi_{1}$ orbital of butadiene, and the formation of the two $\sigma$ bonds of cyclohexene is contributed by both butadiene and ethylene. On the other hand, in the $\mathrm{W}-\mathrm{H}$ diagram, ${ }^{5,6}$ the $\chi_{1}$ orbital of butadiene is connected with the $\sigma_{1}$ orbital of cyclohexene, and the $\pi$ orbital of ethylene with the $\pi$ orbital of cyclohexene in order not to violate the noncrossing rule. In this case, the corresponding electronic formula is expressed by Scheme 2, in which the $\pi$ bond of cyclohexene is formed by the $\pi$ bond of ethylene, and the formation of the two $\sigma$ bonds of cyclohexene is due to only butadiene. According to FO theory, ${ }^{2}$ the $\chi_{2}$ orbital of butadiene (HOMO; the highest occupied molecular orbital) should interact with the $\pi^{*}$ orbital of ethylene (LUMO; the lowest unoccupied molecular orbital), and the $\chi_{3}{ }^{*}$ of butadiene (LUMO) with the $\pi$ of ethylene (HOMO). These correlations apparently correspond to Scheme 1, though FO theory can only describe the correlation between the frontier orbitals. 
It should also be added that Scheme 1 has been reported to be most appropriate for a simplistic representation of the mechanism of the Diels-Alder reaction. ${ }^{7}$

In 1999, Patterson has pointed out in his pedagogical paper on the $\mathrm{W}-\mathrm{H}$ rules that it is incorrect to interpret orbital correlations as a physical mapping or connection of specific reactant orbitals to particular product orbitals. ${ }^{48}$ Our standpoint is totally different from the Patterson's. That is, our dynamic correlation diagrams make it possible to interpret orbital correlations as a physical mapping or the above connections. Obviously, the diagrams which cannot indicate any physical mapping or the connections are meaningless.

\subsection{The interconversions between hexatriene and cyclohexadienes}

Another typical example is the interconversions between hexatriene and cyclohexadienes. The symmetry-allowed photochemical conrotatory cyclization of cis-hexatrienes and the reverse reaction were of great importance in studies on vitamin D. $\mathrm{W}-\mathrm{H}$ rules were first devised to explain these electrocyclic reactions. Although $\mathrm{W}-\mathrm{H}$ rules have succeeded in explaining the stereospecificity of these reactions, not a single correlation diagram has been drawn by Woodward and Hoffmann. Figure 3

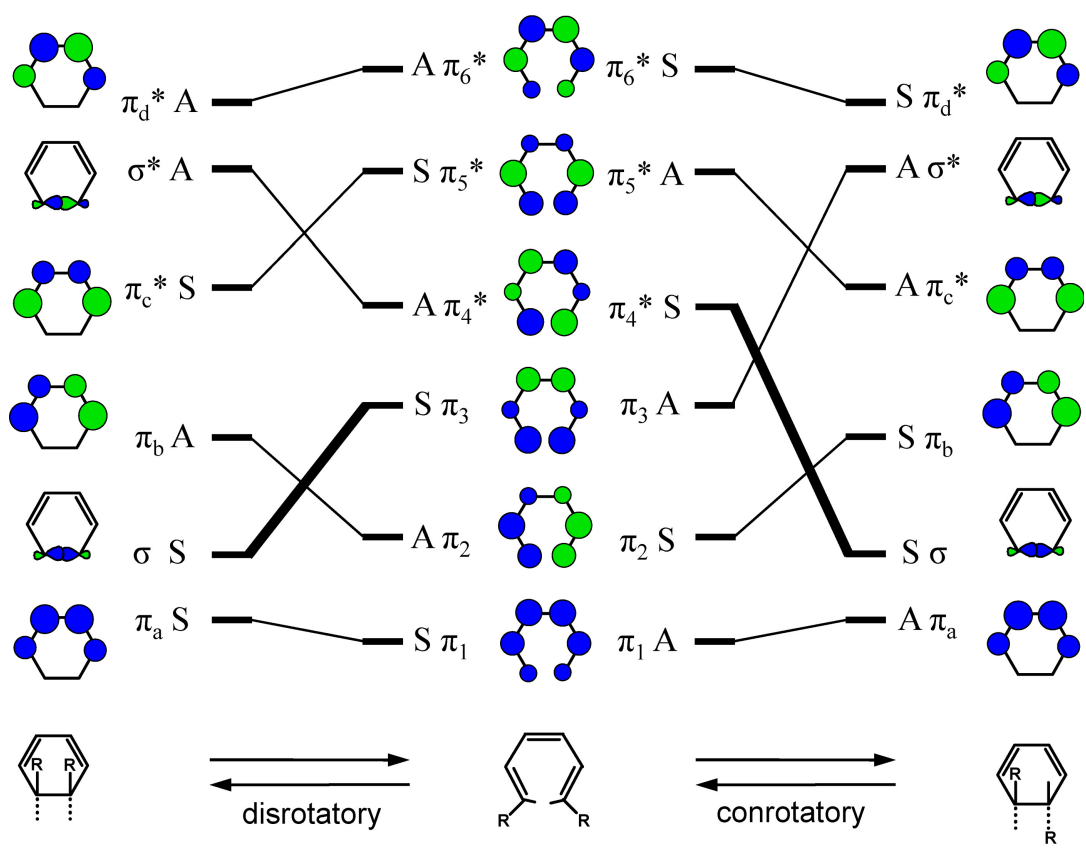

Fig. 3. A dynamic correlation diagram for interconversions between hexatriene and cyclohexadienes. In this diagram, the energy levels of orbitals are slightly different from those in the diagram by Lowry and Richardson. ${ }^{49}$ In the present diagram, the energy level of a $\mathrm{C}-\mathrm{C} \sigma$ bond was taken as $1.3 \beta$ ( $\beta=$ the value of the overlap integral). Since the bonding energies of a $\mathrm{C}-\mathrm{C}$ single bond and a $\mathrm{C}=\mathrm{C}$ double bond are 83 and $147 \mathrm{kcal} \mathrm{mol}^{-1}$, respectively, the bonding energy for the $\pi$ bond of the double bond should be approximately $64(=147-83) \mathrm{kcal} \mathrm{mol}^{-1}$. This value corresponds to $\beta$ obtained by the Hückel method. Thus, the energy level of a $\mathrm{C}-\mathrm{C} \sigma$ bond becomes $1.3 \beta(=83 / 64)$. 
depicts a dynamic correlation diagram according to the principle of minimum deformation of orbital phases. The disrotatory pathway is thermally allowed because there is no correlation line connecting a bonding orbital and an antibonding orbital. By contrast, the conrotatory pathway is thermally forbidden and only photochemically allowed because there is a correlation between $\pi_{4}{ }^{*}$ of hexatriene and $\sigma$ of cyclohexadiene. In the conrotatory reaction, there is a "crossing" of two correlation lines connecting the electronic states of the same symmetry. This diagram is consistent with FO theory because the $\sigma$ orbitals of cyclohexadienes, which are newly formed (or cleaved), are related to the frontier orbitals of hexatriene, as indicated by the thick lines. It should be reemphasized that FO theory can only explain the correlation between the frontier orbitals. Our diagram, on the other hand, shows all the correlations between all the orbitals. Of course, this diagram agrees with $\mathrm{W}-\mathrm{H}$ rules (but not with $\mathrm{W}-\mathrm{H}$ 's correlation diagram method). ${ }^{5,6}$ Furthermore, recent studies $^{28}$ have yielded correlations similar to ours by using the complicated treatments of "avoided crossing" or "conical intersection." It may be worth noting that Lowry and Richardson ${ }^{49}$ have proposed the diagram to which the noncrossing rule is applied. Unfortunately, their diagram disagrees with FO theory in that the $\sigma$ orbitals of cyclohexadienes are not related to the frontier orbitals of hexatriene.

\subsection{The electrophilic substitution of naphthalene}

We must look at one further important example. It is well known that FO theory was initially developed to explain the electrophilic or nucleophilic reaction of naphthalene. However, FO theory can explain only the correlation between the frontier orbitals. Clearly, $\mathrm{W}-\mathrm{H}$ theory has no power over these reactions because they are not pericyclic reactions, and there is no orbital symmetry. If the noncrossing rule is applied to these reactions, the orbitals of the reactant must be simply connected with those of the product from the bottom level up, which is obviously meaningless. To the best of our knowledge, no one has yet succeeded in explaining the complete correlation of electronic states for these reactions. Figure 4 shows a dynamic correlation diagram for the electrophilic substitution of naphthalene drawn according to our new concept. Here, the unoccupied MO of naphthalene has not been shown because it does not take part in the reaction. The selection of the orbitals that are more similar to each other is not difficult task by means of usual pattern recognition criterion. If one needs a theoretical approach, by calculating the overlapping integral between the electronic states before and after the reaction, it is possible to find out the combination in which the integral becomes the maximum. The correlation indicated by thick lines apparently shows that HOMO of naphthalene interacts with the electrophile, which is compatible with FO theory. Moreover, this diagram, which is simply drawn to minimize the deformation of the shape of molecular orbitals, explains all correlations of the orbitals. The most fascinating feature of the dynamic correlation diagram theory is its universal applicability: It is applicable to all elementary reactions regardless of their orbital symmetry. 


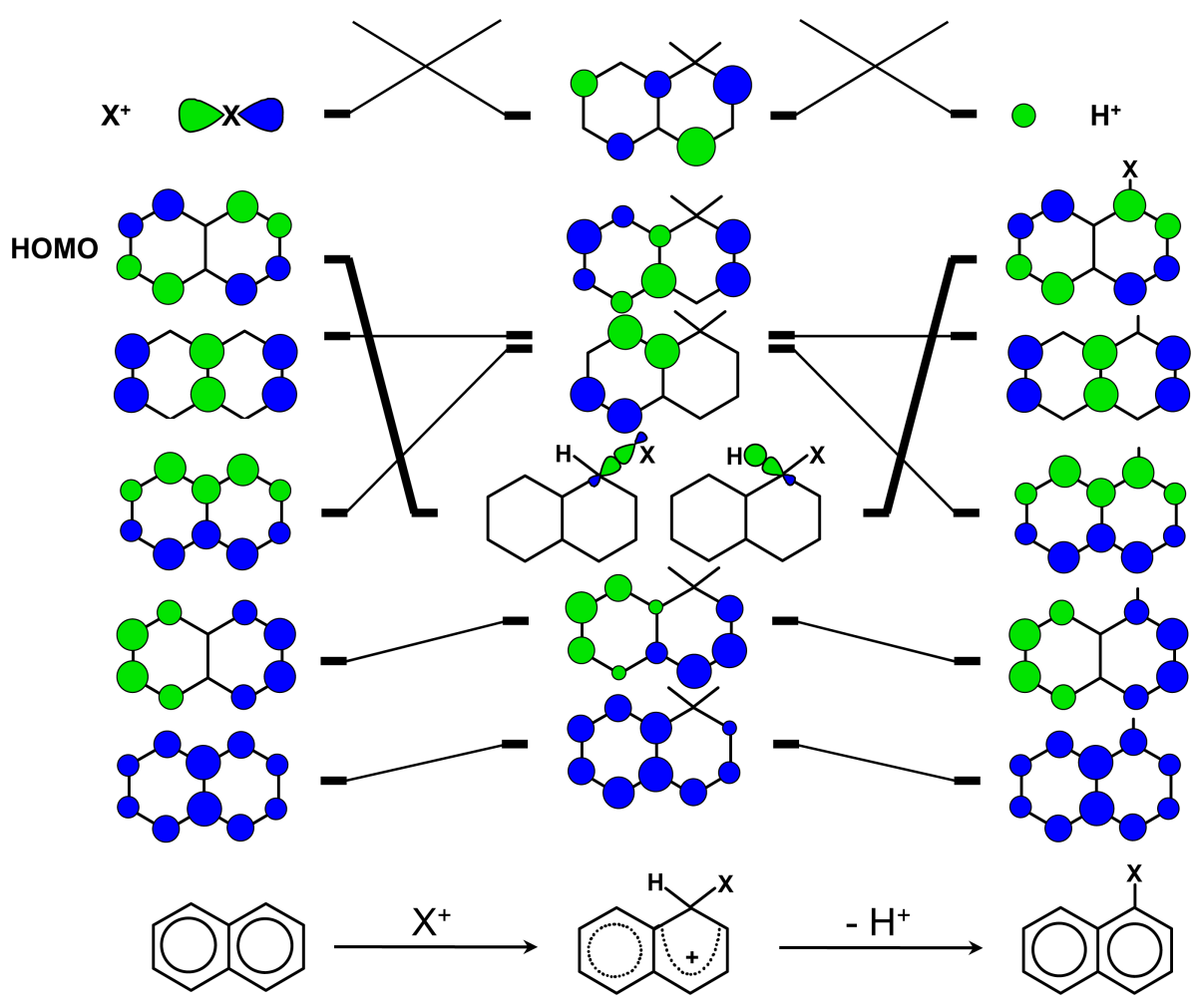

Fig. 4. A dynamic correlation diagram for the electrophilic substitution of naphthalene. HOMO of naphthalene interacts with the electrophile (drawn with thick lines), which is compatible with FO theory. Unoccupied MO of naphthalene has not been shown.

\section{Conclusion}

It has been more than 40 years since FO theory and $\mathrm{W}-\mathrm{H}$ theory was first held in high esteem. Nevertheless, there is the criticism that FO theory cannot explain how molecular orbitals transform from the reactants to products except for the frontier orbitals. ${ }^{50}$ The $\mathrm{W}-\mathrm{H}$ 's correlation diagram method is not as widely applicable as other methods, such as the FO method ${ }^{1,2}$ or Möbius-Hückel method, ${ }^{51}$ as Smith and March have pointed out. ${ }^{52}$ This limitation is well illustrated by the fact that only a limited number of diagrams that are consistent with both FO theory and the experimental results have been drawn so far, even though correlation diagrams are the foundation of $\mathrm{W}-\mathrm{H}$ theory. As a result, the $\mathrm{W}-\mathrm{H}$ 's correlation diagram method has seen no further development to this day. The liberation from the noncrossing rule enables us to unify FO theory and $\mathrm{W}-\mathrm{H}$ theory to our new, universally applicable "dynamic correlation diagram method" based on the principle of minimum deformation of orbital phases. Furthermore, understanding the inherent uncertainty in the energy levels for the midway state of chemical reactions naturally leads to the concepts of stable molecule and elementary reaction, which will deepen 
understanding of chemical reactions and open up a new approach to the study of quantum chemical dynamics.

\section{Acknowledgments}

We would like to thank Prof. Roald Hoffmann (Cornell University) for his warm encouragement concerning this work. We also wish to thank Prof. Keiji Morokuma (Emory University and Kyoto University) and Prof. Emeritus Hiroki Nakamura (Institute for Molecular Science, Japan) for useful discussions.

\section{References}

1. Fukui K, Yonezawa T, Shingu H, J Chem Phys 20:722, 1952.

2. Fukui K, Acc Chem Res 4:57, 1971.

3. Woodward RB, Hoffmann R, J Am Chem Soc 87:395, 1965.

4. Hoffmann R, Woodward RB, J Am Chem Soc 87:2046, 1965.

5. Woodward RB, Hoffmann R, Angew Chem Int Ed 8:781, 1969.

6. Woodward RB, Hoffmann R, The Conservation of Orbital Symmetry, Verlag-Academic, Weinheim, Germany, 1970.

7. Karadakov PB, Cooper DL, Gerratt J, J Am Chem Soc 120:3975, 1998.

8. Hund F, Z Phys 40:742, 1927.

9. Mulliken RS, Phys Rev 32:186, 1928.

10. Dewar MJS, The Molecular Orbital Theory of Organic Chemistry, Mc Graw-Hill Book Company, pp. 331-335, 1969.

11. Nohira H, Tetrahedron Lett 2573, 1974.

12. Hosoya H, Kagaku no ryoiki 28:237, 1974 (in Japanese).

13. Townshend RE, Ramunni G, Segal G, Hehre WJ, Salem L, J Am Chem Soc 98:2190, 1976.

14. von Neuman J, Wigner E, Z Physik 30:467, 1929.

15. Nohira H, Kagaku no ryoiki 28:240, 1974 (in Japanese).

16. Nohira H, Presented at the Third International Congress of Quantum Chemistry 30-IV-3, Kyoto, 1979 and at the 41st IUPAC World Chemistry Congress S05O02, Torino, Italy, 2007.

17. Born M, Oppenheimer R, Ann Phys 84:457, 1927.

18. Heisenberg W, Z Physik 43:172, 1927.

19. Zhu C, Nakamura H, J Chem Phys 97:8497, 1992.

20. Nakai H, Int J Quant Chem 107:2849, 2007.

21. Takatsuka K, Int J Quant Chem 109:2131, 2009.

22. Millie P, Bull Soc Chim Fr 4031, 1966.

23. Devaquet A, Sevin A, Bigot B, J Am Chem Soc 100:2009, 1978.

24. Sevin A, Chaquin P, J Org Chem 47:4145, 1982.

25. Schipper PE, Symmetry and Topology in Chemical Reactivity, World Scientific, Singapore, 1994.

26. Yarkony DR, J Chem Phys 114:2601, 2001.

27. Yarkony DR, J Phys Chem A 105:6277, 2001.

28. Asano Y, Murakami A, Kobayashi T, Goldberg A, Guillaumont D, Yabushita S, Irie M, Nakamura S, J Am Chem Soc 126:12112, 2004.

29. Mori T, Kato S, Chem Phys Lett 476:97, 2009. 
30. Polli D, Altoe P, Weingart O, Spillane KM, Manzoni C, Brida D, Tomasello G, Orlandi, G, Kukura P, Mathies RA, Garavelli M, Cerullo G, Nature 467:440, 2010.

31. Schrödinger E, Ann Phys 384:361, 1926.

32. Schrödinger E, Naturwissenschaften 23:807, 1935 (in German).

33. Schrödinger E, Naturwissenschaften 23:823, 1935 (in German).

34. Schrödinger E, Naturwissenschaften 23:844, 1935 (in German).

35. Schiff LI, Quantum Mechanics, 3 rd ed., McGraw-Hill Inc., pp. 30-31, 1968.

36. Teller E, J Phys Chem 41:109, 1937.

37. Herzberg G, Longuet-Higgins HC, Disc Faraday Soc 35:77, 1963.

38. Longuet-Higgins HC, Abrahamson EW, J Am Chem Soc 87:2045, 1965.

39. Gilchrist TL, Storr RC, Organic Reactions and Orbital Symmetry, Cambridge University Press, 1972.

40. Gill GB, Willis MR, Pericyclic Reactions, Chapman and Hall Ltd., 1974.

41. Pearson RG, Symmetry Rule for Chemical Reactions, Orbital Topology and Elementary Processes, John Wiley \& Sons Inc., 1976.

42. Halevi EA, Orbital Symmetry and Reaction Mechanism, The OCAMS View, SpringerVerlag, 1992.

43. Fleming I, Pericyclic Reactions, Oxford University Press, 1999.

44. Zewail AH, J Phys Chem 100:12701, 1996.

45. Fano U, Lichten W, Phys Rev Lett 15:627, 1965.

46. Streitwieser A Jr, Molecular Orbital Theory for Organic Chemist, John Wiley \& Sons Inc., pp. 33-62, 1961.

47. Rauk A, Orbital Interaction Theory of Organic Chemistry, 2nd ed., Wiley Interscience, New York, 2001.

48. Patterson RT, J Chem Ed 76:1002, 1999.

49. Lowry TH, Richardson KS, Mechanism and Theory in Organic Chemistry, Harper \& Row, Publishers, pp. 581-596, 1976.

50. Bellamy AJ, An Introduction to Conservation of Orbital Symmetry, A Programmed Text, Longman Group Ltd., p. 18, 1974.

51. Zimmerman HE, Acc Chem Res 4:272, 1971.

52. Smith MB, March J, March's Advanced Organic Chemistry, Reactions, Mechanisms, and Structure, John Wiley \& Sons Inc., p. 1068, 2001. 